\title{
A Quantum Field Theoretic Description of Linking Numbers and Their Generalization
}

\author{
Gary T. Horowitz and Mark Srednicki \\ Department of Physics, University of California, Santa Barbara, CA 93106, USA
}

\begin{abstract}
It is shown that the linking number of two surfaces of dimension $p$ and $n-p-1$ in an $n$ dimensional manifold has a natural description in terms of the two-point function of a simple topological field theory. The two-point function of a more general theory (whose partition function yields the Ray-Singer torsion) provides a definition for a generalized linking number.
\end{abstract}

\section{Introduction}

The language of quantum field theory provides a natural framework for describing a variety of results in mathematics. These include the Donaldson invariants of four manifolds [1], the Floer cohomology groups of three manifolds [1], and the Jones polynomials of knot theory [2]. The key idea, is all cases, is to construct a field theory which is independent of a spacetime metric. There are thus no local excitations and the only observables are topological invariants.

The results mentioned above refer to the topology of low dimensional manifolds. Although in many respects this is the richest case to consider, it is of interest to note that there exists a quantum field theoretic description of topological invariants in higher dimensions as well. In fact, the first topological quantum field theory $[3,4]$ was constructed (over ten years ago) to reproduce the Ray-Singer analytic torsion [5]. A basic topological invariant in higher dimensional manifolds is the linking number of a $p$ and $n-p-1$ dimensional submanifold. We will show that this linking number arises naturally from the two-point function of a simple topological field theory. This theory can be viewed as a special case of the theory which yields the Ray-Singer torsion. We will also consider this extended theory and find that the two-point function yields a generalization of the linking number. This is one example of the new insight that the quantum field theoretic approach can bring to topology.

We begin by reviewing the definition of the linking number [6]. Let $M$ be a compact, oriented $n$ dimensional manifold (without boundary). Let $U$ and $V$ be nonintersecting oriented submanifolds of dimension $p$ and $n-p-1$. We assume $U$ and $V$ are homologically trivial, i.e., they are the boundaries of 
surfaces of one higher dimension. Let $V=\partial W$. Then $U$ and $W$ will generically intersect in a finite number of points $p_{i}$. At each $p_{i}$ the orientations on $U$ and $W$ can be combined to yield an orientation on $M$. Define $\operatorname{sign}\left(p_{i}\right)=1$ if the resulting orientation agrees with the original orientation on $M$ and $\operatorname{sign}\left(p_{i}\right)=-1$ otherwise. The linking number of $U$ and $V$ is defined to be

$$
L(U, V) \equiv \sum_{i} \operatorname{sign}\left(p_{i}\right)
$$

Can the assumption that $U$ and $V$ are homologically trivial be relaxed? At first sight this appears likely since the above definition seems to only require that $V$ be trivial. However it is easy to show that if $U$ is nontrivial, then there are topologically inequivalent choices of $W$ (all with $\partial W=V$ ) which yield different answers for the linking number. Mathematically, this is because one can add the Poincare dual of $U$ to $W$. To illustrate this, consider the case of $M=S^{2} \times S^{1} \times S^{1}$. Let $U$ be a two sphere and $T$ be a torus which intersect at one point $p$. Let $V$ be a small circle in $T$ surrounding $p$. Then one choice of $W$ is the small disk in $T$ containing $p$ which yields a linking number of one. But another choice of $W$ is the complement of the disk in the torus which yields a linking number of zero.

If $U$ and $V$ are two circles in $R^{3}$, then there is a well known integral formula for the linking number due to Gauss:

$$
L(U, V)=\frac{1}{4 \pi} \int_{U} d x_{i} \int_{V} d y_{j} \varepsilon^{i j k} \partial_{k}|x-y|^{-1},
$$

where $|x-y|$ denotes the standard Euclidean distance between $x$ and $y$. This formula is easily generalized to surfaces of dimension $p$ and $n-p-1$ in $R^{n}$ by replacing $(4 \pi)^{-1}|x-y|^{-1}$ by $\Gamma(n / 2)\left[(2 n-4) \pi^{n / 2} p !(n-p-1) !\right]^{-1}|x-y|^{2-n}$ and using the $n^{\text {th }}$ rank $\varepsilon$ tensor (see, e.g., [7]). This generalization also applies to the linking of surfaces in an arbitrary manifold provided that the surfaces are contained in a region diffeomorphic to $R^{n}$. Since the surfaces must be homologically trivial one might expect that this includes all surfaces of interest. However this is not the case. Any surface which is homotopically non-trivial cannot be contained in a region diffeomorphic to $R^{n}$. Some examples of surfaces which are homotopically non-trivial but homologically trivial are the generators of the first homotopy group of a homology sphere. ${ }^{1}$

In Sect. 2 we consider the following topological field theory [8]. Let $B$ be a $p$ form and $C$ be a $n-p-1$ form on an $n$ dimensional manifold $M$. The action is simply

$$
S=\int_{M} B \wedge d C
$$

where $\wedge$ denotes the wedge product and $d$ denotes the exterior derivative. Since the integrand is an $n$ form, the integral is well defined without extra structure such

1 A homology sphere is a manifold with the same homology groups as the sphere 
as a metric or a volume form. Classically, the equations of motion are $d B=0$ and $d C=0$. The action is invariant under diffeomorphisms as well as the gauge transformations $B \rightarrow B+d v, C \rightarrow C+d w$, where $v$ and $w$ are $p-1$ and $n-p-2$ forms respectively. Thus the space of gauge inequivalent solutions is just the space of closed forms modulo the exact forms. In other words, it consists of the de Rham cohomology groups $H^{p}(M) \times H^{n-p-1}(M)$. In ref. [8] canonical quantization was carried out for theories of this type assuming $M=\Sigma \times R$. The physical states were found to be square integrable functions on $H^{p}(\Sigma)$.

Now let $M$ be compact (without boundary) and let $U$ and $V$ be homologically trivial surfaces of dimension $p$ and $n-p-1$ respectively. Consider the two-point function

$$
\left\langle\int_{U} B \int_{V} C\right\rangle=\frac{\int[d B][d \mathrm{C}] \int_{U} B \int_{V} C e^{i S}}{\int[d B][d C] e^{i S}} .
$$

Since the expression inside the expectation value is clearly gauge invariant and metric independent, the result appears to be a topological invariant of the surfaces $U$ and $V$. But one might worry that the measure in the functional integral will depend on a choice of metric so the result may have anomalous metric dependence. It turns out that this is not the case. We will show that the right-hand side is in fact metric independent and equal to $i$ times the linking number of $U$ and $V$ :

$$
\left\langle\int_{U} B \int_{V} C\right\rangle=i L(U, V)
$$

(The factor of $i$ is a standard field theoretic consequence of the $e^{i s}$ weighting.)

The action (1.3) has a natural generalization which we discuss in Sect. 3. Let $G$ be a Lie group and $A$ a flat $G$ connection on $M$. Let $\mathscr{B}$ be a $p$ form transforming in a nontrivial representation of $G$ and let $\mathscr{C}$ be a $n-p-1$ from transforming in the dual representation. We consider the action

$$
S_{A}=\int_{M} \mathscr{B} \wedge D \mathscr{C},
$$

where $D$ denotes the covariant curl: $D=d+A$. Schwarz has shown $[3,4]$ that the partition function for this theory is precisely the Ray-Singer torsion of $M$ with the representation of $\pi_{1}(M)$ given by the holonomy of $A$. In light of Eq. (1.5), it is of interest to consider the two-point function of this theory. Since $\mathscr{B}$ and $\mathscr{C}$ transform under nontrivial representations of the group $G$, their integrals over $U$ and $V$ are not gauge invariant. To obtain a gauge invariant expression, we must include Wilson lines connecting points of $U$ to $V$. Thus we are led to consider

$$
\left\langle\int_{U} \mathscr{B}(x) \int_{V} P \exp \left(\int_{x}^{y} A\right) \mathscr{C}(y)\right\rangle
$$

where $P$ denotes path ordering. This expression now depends not only on $U$ and $V$ but also on the flat connection $A$ and a family of curves $\{\gamma\}$ from $U$ to $V$ used to define the Wilson lines. Under certain conditions which will be discussed in 
Sect. 3, the functional integral is finite and metric independent. Furthermore, the dependence on $\{\gamma\}$ will be shown to reduce to a single curve $\gamma_{0}$ from a point of $U$ to a point of $V$. Expression (1.7) can thus be viewed as providing a definition of generalized linking number $L\left(U, V, A, \gamma_{0}\right)$. In most cases it can be evaluated to yield

$$
L\left(U, V, A, \gamma_{0}\right)=\sum_{i} \operatorname{sign}\left(p_{i}\right) \operatorname{Tr} P \exp \left(\oint_{\gamma_{i}} A\right),
$$

where the sum is over the points $p_{i}$ of intersection of $U$ and a surface $W$ with $\partial W=V$, and $\gamma_{i}$ are certain closed curves passing through $p_{i}$ which are determined by $\gamma_{0}$. If the holonomy is trivial, one recovers the usual linking number (1.1) (times the dimension of the representation). We will see that the generalized linking number is also well defined in some cases where the ordinary linking number is not.

\section{Linking Number}

In this section we will derive Eq. (1.5) relating the two-point function of the topological field theory (1.3) to the linking number. To do this we will need to introduce a Riemannian metric and use some results about the eigenforms of the Laplacian. We therefore begin by reviewing these results.

After introducing a metric on $M$, one has the Hodge dual $*$ which maps $p$ forms to $n-p$ forms and satisfies $* *=(-1)^{p(n-p)}$. There is a natural inner product on $p$ forms:

$$
\left\langle B \mid B^{\prime}\right\rangle=\int_{M} B \wedge * B^{\prime}
$$

The adjoint of $d$ is $\delta=(-1)^{p n+n+1} * d *$ and satisfies $\delta^{2}=0$. The Laplacian on forms is defined to be $\Delta=\delta d+d \delta$. The zero modes of $\Delta$ are called harmonic forms, and there is one harmonic $p$ form for each nontrivial element of $H^{p}(M)$. The Hodge decomposition theorem states that the space of all $p$ forms can be decomposed into the direct sum of three orthogonal subspaces via

$$
B=B^{0}+d \varphi+\delta \chi,
$$

where $B^{0}$ is harmonic, and $\varphi$ and $\chi$ have rank $p-1$ and $p+1$ respectively. Following physicists' terminology, we will refer to $d \varphi$ and $\delta \chi$ as the longitudinal and transverse parts of $B$. Since $d^{2}=0$ and $\delta^{2}=0$, the Laplacian leaves each subspace invariant.

Fix an eigenvalue $\lambda^{2}$ of $\Delta$ and let $\Lambda_{p}$ denote the space of rank $p$ eigenforms of the Laplacian with this eigenvalue. Thus every element of $\Lambda_{p}$ satisfies

$$
\Delta B=\lambda^{2} B \text {. }
$$

By the above remarks, if $\lambda \neq 0$, the space $\Lambda_{p}$ decomposes into the direct sum of the transverse $\left(\Lambda_{p}^{\mathrm{T}}\right)$ and longitudinal $\left(\Lambda_{p}^{\mathrm{L}}\right)$ eigenforms. There are three simple relations between these eigenforms. First, the Hodge dual maps $p$ forms with eigenvalue $\lambda^{2}$ to $n-p$ forms with the same eigenvalue. So it provides an isomorphism form $\Lambda_{p}$ to $\Lambda_{n-p}$. In particular, this implies that det $\Delta_{p}=\operatorname{det} \Delta_{n-p}$, 
where $\Delta_{p}$ denotes the Laplacian on $p$ forms. The other two relations are less well known but equally simple. Let $B$ be an element of $\Lambda_{p}^{\mathrm{L}}$. Set $C=\delta B$. Then clearly $\delta C=0$ and $\Delta C=\delta d C=\delta d \delta B=\lambda^{2} C$ using (2.3). Thus $\delta$ is a map from $\Lambda_{p}^{\mathrm{L}}$ to $\Lambda_{p-1}^{\mathrm{T}}$. Similarly, if $B$ is an element of $\Lambda_{p}^{\mathrm{T}}$, set $C=d B$. Then $d C=0$ and $\Delta C=d \delta C=d \delta d B=\lambda^{2} C$, so $d$ maps $\Lambda_{p}^{\mathrm{T}}$ to $\Lambda_{p+1}^{\mathrm{L}}$. The above relations imply that $\lambda^{-1} * d$ is a linear map from $\Lambda_{p}^{\mathrm{T}}$ to $\Lambda_{n-p-1}^{\mathrm{T}}$. But this operator is also invertible. Indeed, its square is the identity operator (up to a possible sign) on $\Lambda_{p}^{\mathrm{T}}$. So $\Lambda_{p}^{\mathrm{T}}$ and $\Lambda_{n-p-1}^{\mathrm{T}}$ are isomorphic. In other words, the values and degeneracies of the (nonzero) eigenvalues of the Laplacian on transverse $p$ forms and $n-p-1$ forms agree. We will use this fact shortly.

Now consider the two-point function of the topological field theory with action $S=\int_{M} B \wedge d C$ :

$$
\langle B(x) C(y)\rangle=\frac{\int[d B][d C] B(x) C(y) e^{i S}}{\int[d B][d C] e^{i S}} .
$$

As we have mentioned, although $S$ is independent of the metric on $M$, to define the measure we will need to introduce a metric. As in the discussion above, we take the metric to be Riemannian. Nevertheless, as indicated in (2.4), we define the partition function as a functional integral of $\exp (i S)$ rather than $\exp (-S)$, the usual prescription when the metric is Riemannian. Since $S$ is not positive definite, we get a well defined answer only if we use $\exp (i S)$. In ordinary field theories, in which the lagrangian density includes an overall factor of $(\operatorname{det} g)^{1 / 2}$, the relation between the coefficient of $S$ and the signature of the metric can be regarded as arising from the factor of $i$ that appears in $(\operatorname{det} g)^{1 / 2}$ when the signature is changed. The action we are considering contains no such factor (indeed no dependence on the metric at all), so changing the signature does not change the coefficient of $S .{ }^{2}$

To evaluate (2.4), we proceed as follows. We can write the measure for $B$ as

$$
[d B]=\left[d B^{\mathrm{T}}\right]\left[d B^{\mathrm{L}}\right]\left[d B^{0}\right],
$$

where $B^{\mathrm{T}}, B^{\mathrm{L}}$, and $B^{0}$ denote the transverse, longitudinal, and harmonic parts of $B$; the measure for $C$ has a similar decomposition. Only the transverse parts of $B$ and $C$ will contribute when integrated over homologically trivial $U$ and $V$, so we need only calculate the two-point function for the transverse parts of the field. The integrals over the longitudinal and harmonic parts of the field just cancel in (2.4) and we obtain

$$
\left\langle B^{\mathrm{T}}(x) C^{\mathrm{T}}(y)\right\rangle=\frac{\int\left[d B^{\mathrm{T}}\right]\left[d C^{\mathrm{T}}\right] B^{\mathrm{T}}(x) C^{\mathrm{T}}(y) e^{i S}}{\int\left[d B^{\mathrm{T}}\right]\left[d C^{\mathrm{T}}\right] e^{i S}} .
$$

Let $B_{n}$ be a basis of transverse eigenforms of the Laplacian, $\Delta B_{n}=\lambda_{n}^{2} B_{n}$ and $\delta B_{n}=0$, normalized so that $\left\langle B_{m} \mid B_{n}\right\rangle=\delta_{m n}$. Set $C_{n}=(-1)^{n-p-1} \lambda_{n}^{-1} * d B_{n}$. Then from the above discussion, $C_{n}$ are also a basis of normalized transverse eigenforms

\footnotetext{
2 We thank Andy Strominger for suggesting this point of view
} 
of the Laplacian. Expand $B=\sum_{n} b_{n} B_{n}$ and $C=\sum_{n} c_{n} C_{n}$. The action is $S=$ $(-1)^{p(n-p)}\langle B \mid * d C\rangle=\sum_{n} \lambda_{n} b_{n} c_{n}$. We define the measure to be $\left[d B^{\mathrm{T}}\right]=\prod_{n} d b_{n}$ and $\left[d C^{\mathrm{T}}\right]=\prod_{n} d c_{n}$. The terms in (2.6) proportional to $B_{m} C_{n}$ for $m \neq n$ are easily seen to vanish. The term proportional to $B_{n} C_{n}$ has a coefficient $i \lambda_{n}^{-1}$. Thus

$$
\left\langle B^{\mathrm{T}}(x) C^{\mathrm{T}}(y)\right\rangle=i \sum_{n} \lambda_{n}^{-1} B_{n}(x) C_{n}(y) .
$$

Now suppose $U$ and $V$ are homologically trivial surfaces in $M$ of dimension $p$ and $n-p-1$ respectively. Let $V=\partial W$. Then

$$
\left\langle\int_{U} B^{\mathrm{T}} \int_{V} C^{\mathrm{T}}\right\rangle=i \sum_{n} \lambda_{n}^{-1} \int_{U} B_{n} \int_{V} C_{n}
$$

But $d C_{n}=\lambda_{n} * B_{n}$, so

$$
\int_{V} C_{n}=\int_{W} d C_{n}=\lambda_{n} \int_{W} * B_{n}
$$

Substituting (2.9) into (2.8) and using $\int_{U} B^{\mathrm{T}}=\int_{U} B$ and $\int_{U} C^{\mathrm{T}}=\int_{V} C$ we get

$$
\left\langle\int_{U} B \int_{V} C\right\rangle=i \sum_{n} \int_{U} B_{n} \int_{W} * B_{n}
$$

The sum is over the transverse eigenforms of the Laplacian. However, since the longitudinal and harmonic eigenforms will not contribute to (2.10), we can extend the sum to include them, and use completeness:

$$
\sum_{n} B_{n}(x) B_{n}(y)+\text { longitudinal + harmonic }=\delta(x, y) I,
$$

where $\delta(x, y)$ is an $n$ dimensional delta function and $I$ denotes the identity operator on $p$ forms. The result is just a sum over the points of intersection with an overall sign depending on the orientation. This is precisely the linking number (times $i$ ). Thus we have obtained the desired result,

$$
\left\langle\int_{U} B \int_{V} C\right\rangle=i L(U, V)
$$

Note that although a metric was chosen to define the functional integral and appeared in intermediate stages of the calculation through the eigenforms and eigenvalues of the Laplacian, the final answer is indeed a topological invariant.

For the special case of $M=R^{n}$, it is easy to see that (2.8) is equivalent to the generalized Gauss formula. We first note that, for any manifold, (2.7) is the Green's function (times $i$ ) for $* d$ restricted to transverse forms. In momentum space, this operator is simply $i \varepsilon^{j} \cdots k_{j}$, where $k$ is the momentum. The inverse is proportional to $i|k|^{-2} \varepsilon^{j} \cdots k_{j}$. Taking the Fourier transform and integrating over the surfaces we recover the generalization of (1.2). 
It is interesting that the functional integral which yields $\left\langle\int_{U} B \int_{V} C\right\rangle$ is well defined under essentially the same conditions that the linking number is well defined: in both cases one must require that $U$ and $V$ be homologically trivial, and in neither case is it necessary to assume anything about the cohomology of $M$ (since the zero modes drop out of the functional integral). The only difference between the two approaches is that the functional integral may depend on boundary conditions for $B$ and $C$ if $\partial M \neq \varnothing$. (The case of $R^{n}$ above is to be viewed as the limit of $S^{n}$ as the radius goes to infinity.) It seems likely that there always exist boundary conditions such that the functional integral yields the linking number, but we will not explore this issue here. There is no analog of this ambiguity in the purely topological approach.

If one tries to evaluate the functional integral when $U$ is homologically nontrivial, then there is always one zero mode $B^{0}$ (corresponding to the chomology class dual to $U$ ) for which $\int_{U} B^{0} \neq 0$. The integral over this mode causes the functional integral to diverge. If one simply defines the functional integral by omitting this zero mode, then the answer is finite but in general metric dependent. This is not surprising since the specific form of the zero mode depends on the metric, so by excluding it one has introduced a metric dependence in the functional integral. However note that the difficulty which arises in the quantum field theory expression for the linking number when $U$ is nontrivial is quite different from the ambiguity that arises in the topological definition.

If $p$ is odd and $n=2 p+1$, one can set $C=B$ and consider the abelian Chern-Simons action $S=\frac{1}{2} \int B \wedge d B$. In this case, one can choose the eigenforms to satisfy $* d B_{n}=\lambda_{n} B_{n}$. The above analysis applies essentially unchanged and one finds that

$$
\left\langle\int_{U} B \int_{V} B\right\rangle=i L(U, V)
$$

where $U$ and $V$ are now both $p$ dimensional surfaces. There is clearly no problem with self linking if $U$ and $V$ are different surfaces.

\section{Generalized Linking Number}

Schwarz has considered the following generalization of the topological field theory discussed in Sect. 2. Let $A$ be a flat connection for a Lie group $G$, and let $\mathscr{B}$ and $\mathscr{C}$ be forms of rank $p$ and $n-p-1$ respectively transforming in nontrivial dual representations of $G$. Mathematically, $\mathscr{B}$ and $\mathscr{C}$ take values in a flat vector bundle $E$ over $M$ with connection $A$. Consider the action $S_{A}=\int_{M} \mathscr{B} \wedge D \mathscr{C}$, where $D=d+A$ is covariant curl. Since $A$ is flat, $D^{2}=0$. The classical field equations are $D \mathscr{B}=0$ and $D \mathscr{C}=0$ and the gauge transformations are $\mathscr{B} \rightarrow B+D v$ and $\mathscr{C} \rightarrow C+D w$. So the space of gauge inequivalent solutions are the cohomology groups of forms with values in $E, H^{p}(M, E) \times H^{n-p-1}(M, E)$. Schwarz has shown that the partition function for this theory is equal to the Ray-Singer analytic torsion. 
We wish to consider the two-point function. As before we must introduce a Riemannian metric to define the functional integral. Many of the results about ordinary forms used in the last section have natural generalizations to forms taking values in $E$. In particular, after introducing a metric on $M$ (and the fibers of $E$ ), we have the Hodge dual $*$ and a natural inner product: $\left\langle\mathscr{B} \mid \mathscr{B}^{\prime}\right\rangle=\int_{M} \mathscr{B} \wedge * \mathscr{B}^{\prime}$. The adjoint of $D$ and the Laplacian $\Delta_{A}$ are defined as before. The zero modes of $\Delta_{A}$ are in one-to-one correspondence with the nontrivial elements of $H^{p}(M, E)$.

The theory of eigenforms of the Laplacian also carries over to forms taking values in a flat vector bundle. In particular, we have a Hodge decomposition theorem analogous to (2.2), completeness of eigenfunctions, and the analog of the result used in the previous section: the values and degeneracies of the eigenvalues of the Laplacian on transverse $p$ and $n-p-1$ forms agree. Let $\mathscr{B}_{n}$ denote a normalized basis of transverse eigenforms of the Laplacian of rank $p$ and set $\mathscr{C}_{n}=(-1)^{n-p-1} \lambda_{n}^{-1} * D \mathscr{B}_{n}$. The evaluation of the two-point function is identical to the one in Sect. 2 and we obtain

$$
\left\langle\mathscr{B}^{\mathrm{T}}(x) \mathscr{C}^{\mathrm{T}}(y)\right\rangle=i \sum_{n} \lambda_{n}^{-1} \mathscr{B}_{n}(x) \mathscr{C}_{n}(y) .
$$

The differences with the theory of Sect. 2 become apparent at the next stage. In order to extract a gauge invariant quantity, we must now pick not only two surfaces $U, V$, but also a family of curves from $U$ to $V$. More precisely, for each point $x \in U$ and $y \in V$ we pick a homotopy class of curves $\gamma$ from $x$ to $y$. We now introduce a family of Wilson lines and consider

$$
\left\langle\int_{U} \mathscr{B}^{\mathrm{T}}(x) \int_{V} P \exp \left(\int_{x}^{y} A\right) \mathscr{C}^{\mathrm{T}}(y)\right\rangle .
$$

Since the connection is flat, the Wilson line depends only on the homotopy class of the curves $\gamma$.

The above expression will in general be metric dependent. In order to avoid this metric dependence, one must insure that the longitudinal and zero modes do not contribute inside the integral, so that the restriction to the transverse parts of the forms in (3.2) can be dropped. (Note that since the action $S_{A}$ only depends on the transverse parts of the forms, (3.2) with the restriction to transverse forms removed will diverge if the longitudinal or zero modes contribute.) In Sect. 2, this was accomplished by requiring that $U$ and $V$ be homologically trivial. In the present case, there are additional conditions. These arise because the Wilson lines $P \exp \left(\int_{x}^{y} A\right)$ may not be continuous in $x$ and $y$. If $U$ contains a closed curve with nontrivial holonomy, then as $x$ goes around the curve, $P \exp \left(\int_{x}^{y} A\right)$ will jump discontinuously. In this case, $\int_{U} P \exp \left(\int_{x}^{y} A\right) D \phi \neq \int_{U} d\left(P \exp \left(\int_{x}^{y} A\right) \phi\right)=0$ due to delta function contributions from the discontinuity. Thus in order that the longitudinal modes not contribute, one must insure that the Wilson lines are continuous in $x$ and $y$. This leads us to the following definition: 
Definition. A surface $U$ is holonomically trivial if every closed curve on $U$ has trivial holonomy.

This condition clearly depends on the connection as well as the surface. It should not be confused with the purely topological conditions of being homotopically trivial (if $U$ can be continuously shrunk to a point) or homologically trivial (if $U$ is the boundary of a surface of one higher dimension). However these conditions are related. If a surface is homotopically trivial, it is always holonomically trivial (for any flat connection). More generally, if every generator of $\pi_{1}(U)$ is homotopically trivial in $M$ then $U$ is holonomically trivial.

Thus the longitudinal modes will not contribute if $U$ and $V$ are holonomically trivial and without boundary. What about the zero modes? These will not contribute if $U$ and $V$ are both homologically trivial and there exist holonomically trivial surfaces $W$ and $W^{\prime}$ with $\partial W=V$ and $\partial W^{\prime}=U$. In this case one can continuously extend the Wilson lines to all points of $W$ and use the fact that for every zero mode $\mathscr{C}^{0}$,

$$
\int_{V} P \exp \left(\int_{x}^{y} A\right) \mathscr{C}^{0}(y)=\int_{W} P \exp \left(\int_{x}^{y} A\right) D \mathscr{C}^{0}(y)=0,
$$

and similarly for the zero modes of $\mathscr{B}$.

Given $U$ and $V$ satisfying the above conditions one can choose the family of curves $\{\gamma\}$ as follows. For simplicity we assume that $U$ and $V$ are path connected. If not, the following procedure can be applied to each connected component. Pick a point $u_{0} \in U$ and another point $v_{0} \in V$. Now connect each point of $U$ to $u_{0}$ with a curve in $U$. Since $U$ is holonomically trivial, the answer will be independent of how these curves are chosen. Similarly, connect each point of $V$ to $v_{0}$. Now pick a curve $\gamma_{0}$ connecting $u_{0}$ to $v_{0}$. This is the only arbitrariness in the choice of curves. The two-point function (3.2) will depend on the homotopy class of $\gamma_{0}$ but only change by an overall factor when this is changed.

If $U$ and $V$ satisfy the above conditions and the curve $\gamma_{0}$ is chosen, then the two-point function

$$
i L\left(U, V, A, \gamma_{0}\right) \equiv\left\langle\int_{U} \mathscr{B}(x) \int_{V} P \exp \left(\int_{x}^{y} A\right) \mathscr{C}(y)\right\rangle
$$

is finite and metric independent. It can thus be viewed as a generalization of the linking number which depends on a flat connection $A$ and a curve $\gamma_{0}$ in addition to the two surfaces $U$ and $V$.

It may appear that the generalized linking number is in fact independent of $A$ since the expression inside the brackets in Eq. (3.4) depends only on $A$ restricted to $U, V$ and $\gamma_{0}$, and the conditions on $U$ and $V$ require that this restricted connection be trivial. However this is not the case. The functional integral depends on $A$ everywhere on $M$ through the action $S_{A}$. This will become clear when we now derive an explicit formula for the generalized linking number.

From (3.1) we have:

$$
\left\langle\int_{U} \mathscr{B}(x) \int_{V} P \exp \left(\int_{x}^{y} A\right) \mathscr{C}(y)\right\rangle=i \sum_{n} \lambda_{n}^{-1} \int_{U} \mathscr{B}_{n}(x) \int_{V} P \exp \left(\int_{x}^{y} A\right) \mathscr{C}_{n}(y) .
$$


Now let $W$ be a holonomically trivial surface with $\partial W=V$. Connect all points in $W$ to $v_{0}$ with a curve in $W$. Then

$$
\int_{V} P \exp \left(\int_{x}^{y} A\right) \mathscr{C}_{n}(y)=\int_{W} P \exp \left(\int_{x}^{y} A\right) D \mathscr{C}_{n}(y)=\lambda_{n} \int_{W} P \exp \left(\int_{x}^{y} A\right) * \mathscr{B}_{n}(y) .
$$

Substituting into (3.5) we again obtain a sum over the transverse eigenforms of the Laplacian. Since the longitudinal and harmonic modes do not contribute, we can extend the sum to include all modes and use completeness. The integral collapses to a sum over the points $p_{i}$ of intersection of $U$ and $W$. The curve in the Wilson line becomes a closed curve passing through $p_{i}$ and the completeness relation says that only the trace enters. We thus obtain the final result:

$$
L\left(U, V, A, \gamma_{0}\right)=\sum_{i} \operatorname{sign}\left(p_{i}\right) \operatorname{Tr} P \exp \left(\oint_{\gamma_{i}} A\right) .
$$

The closed curve $\gamma_{i}$ starts at $p_{i}$, follows a curve in $U$ to $u_{0}$, then $\gamma_{0}$ to $v_{0}$, and finally a curve in $W$ back to $p_{i}$. The holonomy around these curves is uniquely determined.

The above calculation shows that the right-hand side of (3.7) is independent of the choice of $W$ provided only that $W$ is holonomically trivial and $\partial W=V$. In particular, it is invariant under continuous deformations of $W$. This can be seen directly. Under such deformations new points of intersection may arise in pairs, or pairs of points $p_{i}$ may disappear. Suppose $W$ can be deformed to $W^{\prime}$, and $W^{\prime}$ has two new points of intersection $p$ and $q$ of opposite sign. The contributions from these new points will cancel in (3.7) only if the holonomies agree. The above construction yields a closed curve through each of these points which differ by a closed curve $\gamma$ that starts at $p$, goes to $q$ along $W^{\prime}$, and then returns to $p$ via $U$. Using only the fact that $U$ and $W^{\prime}$ are holonomically trivial, we cannot conclude that this closed curve has trivial holonomy. But we also know that $W^{\prime}$ can be continuously deformed to $W$. Under this deformation the closed curve $\gamma$ shrinks to zero. Since $\gamma$ is homotopically trivial, it is also holonomically trivial and the two contributions to (3.7) cancel.

An example will help to illustrate the generalized linking number. Figure 1 shows two circles $U$ and $V$ in $R^{3}$ which have ordinary linking number zero. We now remove the two dotted vertical lines and put on a $U(1)$ connection with holonomy $e^{i \alpha}$ around one line and $e^{-i \alpha}$ around the other. Then $U$ and $V$ are both holonomically trivial and without boundary. Figure 1 also shows a curve $\gamma_{0}$ connecting a point $u_{0} \in U$ to $v_{0} \in V$. Let $W$ be the disk shown. It is also holonomically trivial. There are two points of intersection $p$ and $q$ which occur with opposite sign. The closed Wilson loop containing $p$ is holonomically trivial but the one containing $q$ is not. The generalized linking number is thus

$$
L\left(U, V, A, \gamma_{0}\right)=1-e^{i \alpha} \text {. }
$$

In this example, $M$ has a boundary, but we have previously assumed that $\partial M=\varnothing$ so that the functional integral would not depend on arbitrary boundary conditions. Fortunately, it is easy to modify this example so that $\partial M=\varnothing$. Imagine slicing Fig. 1 with a family of horizontal planes. Each plane has the topology of $R^{2}$ with two points removed. Now add a point at infinity and a handle to connect 


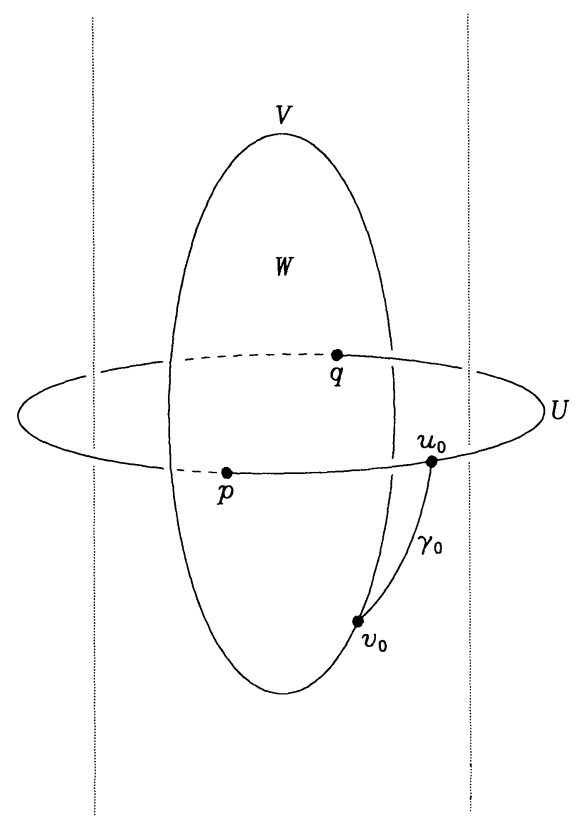

Fig. 1. An example of the generalized linking number is shown. $U$ and $V$ are circles with ordinary linking number zero. The two verticle lines are removed and a $U(1)$ connection $A$ (not shown) is added with holonomy $e^{i \alpha}$ around one line and $e^{-i \alpha}$ around the other. The generalized linking number is $L\left(U, V, A, \gamma_{0}\right)=1-e^{i \alpha}$

the boundaries of the two removed points. The resulting surface has the topology of a torus $T^{2}$. Finally, identify the top and bottom surface to obtain $T^{3}$. We now have an example of two circles in a three torus with generalized linking number given by (3.8).

The two-point function (3.4) and hence the generalized linking number is actually well defined under certain conditions more general than we have discussed so far. In particular, it can be defined in some cases when the ordinary linking number can not. For example, suppose $H^{p}(M, E)=0$ and $H^{n-p-1}(M, E)=0$ so there are no zero modes. Then we do not have to require that $U$ and $V$ be homologically trivial. (We must still require that they are holonomically trivial so the longitudinal modes drop out. Thus it again suffices to pick a single curve $\gamma_{0}$ from $U$ to $V$ to define the Wilson lines.) In the theory of Sect. 2, if there are no zero modes then all surfaces are homologically trivial. But that is not the case in the presence of a flat connection.

Consider the example mentioned in the introduction where $M=S^{2} \times T^{2}, U$ is a nontrivial two sphere, $T$ is a torus intersecting $U$ at one point $p$, and $V$ is a small circle in $T$ surrounding $p$. If we add a connection with nontrivial holonomy around both generators of the torus, then there will in general be no zero modes. Since $U$ and $V$ are holonomically trivial, the generalized linking number is unambiguously defined. If we choose $\gamma_{0}$ to be a short curve from the point $p \in U$ to a point $v_{0} \in V$, then it equals $\operatorname{Tr} 1$, which is the dimension of the representation. 
This can be seen by letting $W$ be the small disk in $T$ containing $p$ and following the argument given earlier. If one had instead chosen the complement of the disk for $W$, then it would not have been holonomically trivial. The functional integral would now have a contribution from the discontinuity in the Wilson lines which exactly compensates for the zero contribution from the usual number.

It is not clear to us what applications the generalized linking number will have. In light of recent connections between the ordinary linking number and fractional spin and statistics (see, e.g. $[9,7]$ ) potential applications to physics seem promising. From a purely mathematical standpoint, the fact that the generalized linking number arises naturally from the same topological field theory that yields the Ray-Singer torsion, and is a straightforward generalization of the ordinary linking number, makes it worthy of further study.

Acknowledgements. It is a pleasure to thank Marty Scharlemann for useful discussions. We also thank Pawel Mazur for bringing reference [4] to our attention. This work was supported in part by NSF Grant No. PHY85-06686 and PHY86-14185, and the Alfred P. Sloan Foundation.

Note added in proof. After this manuscript was completed, we received a copy of a preprint by $\mathrm{M}$. Blau and G. Thompson ("Topological Gauge Theories of Antisymmetric Tensor Fields", Trieste preprint SISSA 39/89/FM) which also discusses the relation between the linking number and the topological field theory of Sect. 2.

\section{References}

1. Witten, E.: Commun. Math. Phys. 117, 353 (1988)

2. Witten, E.: Quantum field theory and the Jones polynomial. Commun. Math. Phys. 121, 351-399 (1989)

3. Schwarz, A. S.: Lett. Math. Phys. 2, 247 (1978)

4. Schwarz, A. S.: Commun. Math. Phys. 67, 1 (1979); Schwarz, A. S., Tyupkin, Yu. S.: Nucl. Phys. B242, 436 (1984)

5. Ray, D. B., Singer, I. M.: Adv. Math. 7, 145 (1971)

6. Rolfsen, D.: Knots and Links, Berkeley C. A.: Publish or Perish 1976

7. Wu Y. S., Zee, A.: Phys. Lett. B207, 39 (1988); Wilczek, F., Zee, A.: in preparation

8. Horowitz, G. T.: Exactly soluble diffeomorphism invariant theories. Commun. Math. Phys. 125, 417 (1989)

9. Wilczek, F., Zee, A.: Phys. Rev. Lett. 51, 2250 (1983); Polyakov, A. M.: Mod. Phys. Lett. A3, 325 (1988); Tze, C. H., Nam, S.: Topological phase entanglements of membrane solitons in division algebra sigma models with a hopf term. Virginia Polytechnic Institute preprint VPI-IHEP-88-10

Communicated by S.-T. Yau

Received May 9, 1989 